\title{
IMMUNOCHROMATOGRAPHIC TEST OVER MICROSCOPY IN DIAGNOSIS OF SEVERE MALARIA IN BANGLADESH
}

\author{
Awwal A ${ }^{1}$, Amin $\mathrm{MR}^{2}$, Hafiz SAMMA 3 ; Rahman $\mathrm{M}^{4}$, Ali $\mathrm{M}^{5}$, Sattar A ${ }^{6}$, RahmanMR $^{7}$, Faiz MA $^{8}$
}

\begin{abstract}
Objective: This study was conducted to determine the advantage of Immunochromatographic test over microscopic peripheral smear in clinically suspected severe malaria.

Methods: Total 106 consecutive clinically diagnosed severe malaria cases were enrolled with patients over 12 years of age irrespective of sex, race, religion and pregnancy from March to August 2002, at one medicine unit of Chittagong Medical College Hospital. The immunochromatographic rapid diagnosis test and microscopy were performed in all
\end{abstract} cases.

Results: Sensitivity and specificity of immunochromatographic Paracheck Pf test was found $91.42 \%$ and $90.65 \%$ in this study. Positive predictive value was $90.56 \%$ and negative predictive value was $91.5 \%$. The sensitivity of microscopy was only $69.8 \%$.

Conclusion: Reliable quality controlled malaria microscopy is difficult to provide in many rural remote areas due to lack of logistics manpower and electricity facility. Rapid diagnostic test like Paracheck Pf test could be of use in such a situation which is reliable, cheap and easy to perform.

Key words: Advantage, immunochromatographic test, microscopic test

\section{Introduction}

Malaria continues to be the world's most important protozoan-parasitic infection. It ranks third among major infectious disease with a mortality rate lower only than pneumococcal respiratory infections and tuberculosis ${ }^{1}$. The annual number of cases of clinical malaria worldwide is estimated to be 300 and 500 million, with 1.5 to 2.7 million deaths ${ }^{2,3}$. Malaria is an important public health problem of Bangladesh with about $80 \%$ of the 125 million population at risk for infection with either Plasmodium falciparum ( $P$ falciparum) or Plasmodium vivax ( $P$ vivax). Transmission of $P$ falciparum is more focal with an estimated 35 million people at risk and about 10 million people at high risk ${ }^{4}$. Strengthening of national capabilities to provide early diagnosis and treatment both within and outside the general health services is of highest priority in WHO's Action Plan for Malaria Control 1995-20005.

Early diagnosis and prompt treatment is of paramount importance to reduce the mortality and morbidity and also for drug resistance associated with it. The clinical diagnosis of severe malaria can be difficult as there is no component of the clinical picture or its complications that are unequivocally diagnostic of the infection. Microscopic analysis for identifying malaria infections has been done for more than a century. Slides obtained from finger prick blood samples when properly stained and skillfully examined, permit species diagnosis, estimations of parasitaemia, identification of the various blood forms of parasite and often, prognosis of the likely course of disease. The technique is capable of accurate and reliable diagnosis when performed by skilled microscopist using defined protocols. These qualifiers, skill of the microscopist and use of proven, defined procedures frequently present the greatest obstacles to fully achieving the potential accuracy of microscopic diagnosis. Conversely patients with severe malaria are occasionally seen whose blood film at the time of presentation is negative because of previous treatment, a highly synchronous infection, or comprehensive sequestration of parasitized erythrocytes in deep vascular beds. Yet the simplest and surest test is the time honoured peripheral smear study for malarial parasites.

However, difficulty in maintaining required technical skills and infrastructure has spurred the development of several non-microscopic malaria rapid diagnostic devices (MRDDs). These tests involve identification of the parasitic antigen or the antiplasmodial antibodies or the parasitic metabolic products. Namely Parasight F test, Optimal Assay, the immunochromatographic test (Paracheck Pf test), Polymerase chain reaction, detection of antibodies by Radio immuno assay, immunofluorescence or enzyme immuno assay. The current generation of MRDDs are based on antigen capture immunoassay methodologies utilizing immunochromatographic strip (ICS) technology. The

1. Dr Anisul Awwal MBBS, FCPS, MD (Card), Assistant Professor of Cardiology, Chittagong Medical College; 2. Dr Mohammad Robed Amin MBBS, FCPS, Assistant Professor of Medicine, Dhaka Medical College, email: robedamin@yahoo.com; 3. Col SAMMA Hafiz MBBS, FCPS, Classified Specialist in Medicine, CMH Dhaka; 4. Dr M Rahman MBBS, FCPS, MD (Gastro), Associate Professor (Gastroenterology) Uttara Medical College, Dhaka; 5. Dr Mohammad Ali MBBS, FCPS, Assistant Professor of Medicine, Bogura Medical college; 6. Dr A Sattar MBBS, FCPS, Assistant Professor of Medicine, Chittagong Medical college; 7. Prof MR Rahman MBBS, FCPS, Professor of Medicine, Shaheed Suhrawardy Medical College, Dhaka; 8. Prof MA Faiz MBBS, FCPS, FRCP, Professor of Medicine (PRL), Sir Salimullah Medical College, Dhaka 
Paracheck Pf (Orchid Biomedical Systems, India) is a rapid self performing qualitative, two site sandwich invitro immunoassay for the detection of circulating $P$ falciparum Histidine rich protein-2 (Pf HRP-2), is a water soluble protein that is released from parasitized erythrocytes and is specific to the $P$ falciparum species $^{6,7}$. The test uses two antibodies specific for Pf HRP-2 antigen. One of the antibodies is attached to visible colloidal gold and impregnated into a sample pad, while the second antibody is immobilized as a line across the test strip. In a positive sample, Pf HRP-2 complexed with the gold labeled antibody is captured by the antibody on the membrane and a pink line forms. In a negative sample, no pink line forms. It is rapid, cost effective and doesn't require specialized equipment. Test can be carried out at the point of contact between the patient and the health care worker ${ }^{8}$.

The reported study was conducted to observe the advantage of immunochromatographic test over microscopic peripheral smear in clinically suspected severe malaria cases.

\section{Materials and Methods}

This prospective study was carried out at the medicine unit of Chittagong Medical College Hospital (CMCH) situated in south-east region of Bangladesh during the period from March 2002 to August 2002. One hundred and six cases of adult clinically suspected severe malaria patients admitted to the hospital were included in the study.

\section{Inclusion Criteria}

$\mathrm{m}$ Clinical syndrome of severe malaria

$\mathrm{m}$ Patients over 12 years of age

$\mathrm{m}$ Irrespective of sex, race, religion and pregnancy.

\section{Exclusion Criteria}

$\mathrm{m}$ Uncomplicated and treatment failure malaria cases

$\mathrm{m}$ Patients/attendants unwilling to give informed consent to take part in the study

$\mathrm{m}$ Patients dying before collecting the blood sample for investigations and recording the information.

All cases admitted to one of the 3 adults' medical units of $\mathrm{CMCH}$ (MU III) fulfilling the inclusion criteria were enrolled. An informed consent was taken from the patients or eligible attendants to take part in the study and to collect blood samples for investigations. Two separate individuals performed the strip test and collection of a blood smear for microscopy on admission. None had any idea about the result of other test. Thick and thin smears were prepared from finger prick, stained with Giemsa stains and examined by an experienced technician initially followed by re-examined and cross checked by a clinical pathologist (trained in WHO Malarial Microscopy). Patients' data were recorded on pre-designed case record form. One hundred and six consecutive cases were recorded. Laboratory investigations to exclude other possible differential diagnosis were also done from available hospital facility and those not available done from locally available private laboratories. Routinely performed investigations include malarial parasite count, Paracheck Pf test (immunonochromatographic test), Blood for Haemoglobin \%, Total count \& Differential count, Erythrocyte sedimentation rate in 1st hour, Platelet count; Peripheral Blood film, Urine routine microscopic examination, Blood urea, Serum creatinine, Chest X-ray and Cerebrospinal fluid study.

The patients were treated according to the National Guidelines i.e. parenteral quinine followed by oral quinine whenever patients could take orally. Dose of quinine was $10 \mathrm{mg} / \mathrm{kg} /$ dose (quinine dihydrochloride) as salt in isotonic fluid (5\% dextrose water/saline) $500 \mathrm{ml}$ over 3-4 hours and repeated 8 hourly. Quinine therapy was continued for a total of 7 days. Other general and specific measures, such as $25 \%$ glucose for suspected/proved hypoglycaemia, blood transfusion for severe anaemia. Intravenous diazepam and anticonvulsant, peritoneal dialysis (for selected patients) were taken when appropriate. Outcome of severe Malaria was categorized as full recovery, recovery with complication, death and others.

\section{Results}

In this study, age range was from 12 to 65 years. Out of 106 cases, 69 (65\%) patients were aged below 30 years (Table I). This study included 83 male $(78.3 \%)$ and 23 $(21.7 \%)$ female patients with ratio of $3.6: 1$. Out of 23 female patients, 5 patients were pregnant. Two of them died and one had abortion. Thirty six patients (34\%) have residence in malaria endemic zone (Table II) and 69 $(55.1 \%)$ patients had history of taking antimalarial drugs before hospitalization.

Table -I: Distribution of patient as per age group $(\mathrm{n}=106)$

\begin{tabular}{|l|c|c|c|}
\hline $\begin{array}{l}\text { Age group } \\
\text { (in years) }\end{array}$ & $\mathbf{n}$ & $\begin{array}{c}\text { Range } \\
\text { (in years) }\end{array}$ & $\begin{array}{c}\text { Mean } \pm \text { SD } \\
\text { (in years) }\end{array}$ \\
\hline$<20$ & 46 & $12-20$ & $17.63 \pm 2.23$ \\
\hline $21-30$ & 23 & $21-30$ & $25.48 \pm 3.03$ \\
\hline $31-40$ & 23 & $31-40$ & $34.45 \pm 4.23$ \\
\hline$\geq 41$ & 14 & $42-65$ & $48.29 \pm 6.21$ \\
\hline
\end{tabular}

Table-II: Malaria related information $(\mathrm{n}=106)$

\begin{tabular}{|l|c|c|}
\hline Parameters & n & \% \\
\hline Residence in endemic zone & 36 & 34.0 \\
\hline History of traveling to malarial area & 56 & 52.8 \\
\hline History of malaria in the past & 28 & 26.4 \\
\hline Antimalarials taken & 69 & 65.1 \\
\hline
\end{tabular}


Table -III: Distribution of patient as per presenting features $(\mathrm{n}=106)$

\begin{tabular}{|l|c|c|}
\hline Feature & $\mathbf{n}$ & $\mathbf{\%}$ \\
\hline Vomiting & 76 & 71.7 \\
\hline Dark-coloured urine & 72 & 67.9 \\
\hline Severe prostration & 71 & 67.0 \\
\hline Rousable coma & 33 & 31.1 \\
\hline Unrousable coma & 30 & 28.3 \\
\hline Oliguria (<400 ml/day) & 18 & 17.0 \\
\hline Anaemia & 71 & 67.0 \\
\hline Jaundice & 57 & 53.8 \\
\hline Hepatomegaly & 73 & 68.9 \\
\hline Splenomegaly & 54 & 51.0 \\
\hline
\end{tabular}

It was observed that patients recovered fully had mean Glasgow coma score (GCS) $11.13 \pm 3.63$; where as 8.88 \pm 2.89 was the mean score for the patient died during treatment. Anaemia, thrombocytopenia, hypoglycemia, mild renal impairment were observed in investigation in this study(Table IV).

Table - IV: Findings of laboratory investigation $(\mathrm{n}=106)$

\begin{tabular}{|l|c|c|}
\hline Variables & Range & Mean \pm SD \\
\hline Haemoglobin $(\mathrm{g} / \mathrm{dl})$ & $3.5-14.10$ & $8.78 \pm 2.49$ \\
\hline $\begin{array}{l}\text { Total count of W.B.C/ } \\
\text { cumm }\end{array}$ & $4000-15000$ & $4607.55 \pm 262.82$ \\
\hline Neutrophil $(\%)$ & $58-76$ & $66.92 \pm 9.84$ \\
\hline Platelet /cu mm & $41,000-150,000$ & $95,000 \pm 18,000$ \\
\hline Blood glucose mg/dl & $50-313$ & $103.57 \pm 47.48$ \\
\hline Serum Creatinine mg/dl & $0.50-8.00$ & $1.37 \pm 1.15$ \\
\hline $\begin{array}{l}\text { Blood film for malarial } \\
\text { parasite }\end{array}$ & $100-25 \times 10^{5}$ & $215.21 \pm 446.74$ \\
\hline
\end{tabular}

Among 106 patents, microscopy was positive in 74 (69.8\%) cases with variable range and Parcheck Pf test was positive in $96(90.6 \%)$ cases. Out of the microscopically positive cases, 73 cases were $P$ falciparum and 1 case was $P$ vivax. Out of those patients Paracheck Pf test was positive in 73 cases. Among 32 (30.2\%) microscopic negative cases, 9 (28.1\%) cases were negative both in malarial microscopy and Parachek Pf test. The other $23(71.9 \%)$ cases were positive for Paracheck Pf test but negative for malarial microscopy. Out of these 23 cases 20 had prior history of antimalarial drug intake before hospitalization.

$\begin{array}{ll}\text { m Sensitivity } & : 91.42 \% \\ \text { m Specificity } & : 90.65 \% \\ \text { m Positive predictive value } & : 90.56 \% \\ \text { m Negative predictive value } & : 91.5 \%\end{array}$

Finally 97 patients (915\%) patents recovered and 9 patents $(8.57 \%)$ patients died. Fifteen patients $(14.2 \%)$ recovered with complication.

\section{Discussion}

One hundred and six adult cases of severe malaria (83 male, 23 female; male-female ratio 3.6:1) were studied during 6 months of the study period, who got admitted in the medicine units of Chittagong Medical College Hospital. Majority of the cases occurred in males of working age group. Out of 23 female patients 5 (21.7\%) were pregnant and 2 of them died and 1 had abortion. It indicates that severe malaria in pregnancy remains a great threat to both mother and fetus.

Thirty six (34\%) patients had residence in endemic zones. Fifty six (52.8\%) patients had history of travel to malarial area within last 8 weeks indicating that travel to endemic area is a risk factor for malaria. Twenty eight (26.4\%) patients had history of malaria in the past. Sixty nine $(65 \%)$ patients had history of taking antimalarial drug before hospitalization, which create confusion in diagnosis by increasing chance of negative malarial microscopy.

Highest temperature recorded in hospital ranges from $98^{\circ}$ to $106^{\circ} \mathrm{F} \quad\left(\right.$ mean $\left.=102.1 \pm 1.59^{\circ} \mathrm{F}\right)$. Hyperpyrexia (temperature $105^{\circ} \mathrm{F}$ ) was present among 5 hospitalized patients, 3 of them died. The other clinical presentations accordingly were vomiting (71\%), severe prostration $(67 \%)$, dark coloured urine $(67.9 \%)$, oliguria $(18 \%)$, deep breathing (21\%), hepatomegaly (68\%), splenomegaly $(51 \%)$, rousable coma $(31 \%)$ and unrousable coma (28.3\%). Patients with low Glasgow come score at presentation during hospitalization was associated with poor outcome. Fifty five percent of patients who died have GCS $<7$. Similar report was also documented in a study by Faiz et al $^{9}$.

Anaemia was found in $67 \%$ patients, among these, 8 patients $(7.5 \%)$ patients had haemoglobin below $5 \mathrm{gm} / \mathrm{dl}$ i.e. severe anaemia. Out of these 8 patients, 2 died which indicate severe anaemia is a poor prognostic factor. Jaundice was present in $57(53 \%)$ of patients. Seven out of 9 patients who died had jaundice. However, death rate was high in patients with associated renal failure $(55 \%)$ and deep breathing (52\%). This is consistent with the findings of acute renal failure in malaria by Chowdhury et $\mathrm{al}^{10}$.

Microscopic peripheral smear study for malarial parasite was positive in $74(69.8 \%)$ patients. Malarial parasite count varies from $100 / \mathrm{cu} \mathrm{mm}$ to $25 \times 10^{5} / \mathrm{cu}$ mm (mean \pm $\mathrm{SD}=215.21 \pm 446.74$ per cumm). Seventy three of these patient's slide show $P$ falciparum, where as 1 patient had $P$ vivax. Immunochromatographic Paracheck Pf test was positive in $96(90.6 \%)$ patients. Paracheck Pf test was positive in all but 1 slide positive malaria cases. The only slide positive case which Paracheck Pf test fail to detect was $P$ vivax. Which is consistent with its principle as Paracheck Pf detect only HRP-2 released from $P$ falciparum infected cells. It can be deduced from here that diagnostic capability of Immunono- 
chromatographic test are superior to microscopy. In an unpublished study by Hossain et al diagnostic capability of malarial microscopy and dipstick antigen capture assay (Paracheck Pf test) was observed 64\% and 65\% respectively.

Among 23 Paracheck Pf positive but malarial slide negative patients, 20 had history of receiving antimalarial drugs prior to hospitalization. In severe falciparum malaria peripheral parasitaemiae may be missed due to parasite sequestration in the microvasculature ${ }^{11}$. But antigenaemia may provide a clue to diagnosis ${ }^{12}$. Moreover prior antimalarial treatment also cause negative malarial microscopy, which can affect the malarial parasite count in peripheral blood film. But prior treatment doesn't affect the result of Paracheck Pf test as it detects the HRP-2 protein not the parasite itself.

Sensitivity and specificity of Paracheck Pf test was $91.42 \%$ and $90.65 \%$ in this study. This is consistent with other study showing sensitivity and specificity of immunochromatographic test $91.6 \%$ and $99.4 \%$ by Banchongaksorn et $\mathrm{al}^{13} ; 84 \%$ and $77 \%$ by Genton et $\mathrm{al}^{14}$; $88 \%$ and $97 \%$ by Humar et $\mathrm{al}^{15}$.

In this study positive predictive value (PPV) was $90.56 \%$ and negative predictive value (NPV) was $91.5 \%$. These are also consistent with similar study showing positive predictive value and negative predictive value $90 \%$ and $94 \%$ by Kilian et $\mathrm{al}^{16} ; 98.7 \%$ and $96.2 \%$ by Banchongaksorn et $\mathrm{al}^{13} ; 85 \%$ and $92 \%$ by Bouchaud et $\mathrm{al}^{7}$. So this test is useful where malarial microscopy is not reliable or even if available and where patients have received antimalarial treatment.

\section{Conclusion}

In contrast to light microscopy, Paracheck Pf test is fast and simple. One test can be completed in less than 15 minutes and many can be done simultaneously, reducing the time required for the results to be made available to support the clinical diagnosis, if accuracy can be assured and the test made affordable for those in need.

Both the specificity and sensitivity of the Paracheck Pf when measured against current "gold standards "are generally around $90 \%$. This is considered to be at least equal to that currently achieved in well organized malaria diagnostic laboratories, and probably well in excess of that achieved in many general health services, particularly those at the periphery.

A potential problem with the Paracheck Pf is that circulating antigen may be detected after elimination of viable parasites from the blood stream. However, these antigenaemic patients were usually clinically asymptomatic after clearance of the parasitaemias. Thus, as treatment should be based on clinical criteria supported by laboratory findings and not on laboratory results alone, this type of false positive should not lead to large numbers of unnecessary treatments. Neither should those false positives that may also result from the presence of immature gametocytes. The assay does not detect mature gametocytes.

\section{Refernces}

1. WHO. The Diagnosis and Management of severe and complicated. Malaria, part-1 learners Guide, 1995. Trial edition. Training unit, Division of Control of Tropical diseases.

2. Krogstad DJ. Malaria, In: Cecil Text book of Medicine 19th edition WB Saunders Company, New Youk. 1992: 1972-1975.

3.Warrell DA. Pathophysiology of severe falciparum malaria. Parasitology. 1987,94:853-76

4. Rahman M.R, Paul DC, Rashid M, Ghosh A. Bangali A.M. Jalil M.A. et. al. A randomized controlled trial on the efficacy of alternative treatment regimens for uncomplicated falciparum malaria in a multidrug resistant falciparum area of Bangladesh - narrowing the options for the National Malaria Control Programme Trans of the R S of Trop. Med. and Hyg. 2001, 95: 661-667

5. White NJ, Breman G. Joel Malaria and Babesiosis : Disease caused by red blood cell parasites in Harrison's Principles of internal medicine 15th edition. edited by Braunwald E, Fauci A, Kasper D. Hauser S.L. Longo DL. Janeseson J.L. et.al. McGraw-Hill; 1,2:1203-1213

6. Wolday D, Balchat, Fessehaye G, Birku Y. Shepherd A, Field trial of the R.T.M. disptick method for the rapid diagnosis of Malaria based on the detection of Plasmodium falciparum HRP-2 antigen in whole blood. Trop. Doc. 2001, 31: 18-21.

7. Bouchaud O, Houze S, Longuet C, Piazza D.P, Ruggieri C, Secardin $\mathrm{Y}$ et al. Use of the parasight-F Diagnostic test for imported malaria in a travel clinic. The Amer. Soc. of Trop. Med. Hyg. 2000, 20: 76-79.

8. WHO: A rapid dipstick antigen capture assay for the diagnosis of falciparum malaria, Based on the report of a joint SEARO/TOR/CTD Informal Consultation on recent advances in diagnostic techniques and vaccines for malaria; Institute of Immunology, New Delhi, India, 1995, 1072: 1-7 CTD/MCM/IM/92-2.

9. Faiz MA, Rahman M R, Hossain M A, Rashid H A.Cerebral Malaria-a study of 104

cases. Bangladesh Med Res Counc Bull 1998, 24:35 42

10.Chowdhury D, Yunus EB, Dutta PK. Acute renal failure in malaria, JCMCTA.1996,7:69-74

11.Warrell D A. Payhophysiology of Severe falciparum malaria. Parasitology. 1987,94:853-76

12. Desakorn V. Silamut K, Angus B, et al. Semi-quantitative measurement of P. falciparum antigen Pf HRP 2 in blood adn plasma. Trams R Soc Trop Med Hyg 1997, 91: 47983

13. Banchongaksorn T, Yomkgul P. Panyium S, Rooney W, Vickers P. A field trial of the parasight $\mathrm{F}$ lest for the diognosis of plasmodium falcipanum infection, trans of the R. soc of trop. Med. and Hyg. 1996, 90: $24-245$

14.Genton B, Paget S. Beck H.P. Gisan M. Alpers Mp. Hii I et. al Dragmsis of plasmdium faciparum infection using para sight $\mathrm{F}$ test in blood and urine of papua new guinean children; Sathiast Asion J. trop. Med pub Health. 1948, 29: 35-37.

15. Humar A, Ohrt c. Harrington M.A. Pillai d, Kain K.C, Para Sight F test compared. with polym,erose chain reaction and microscopy for the diagnosis of plasmodium falciparum malaria in travelers. The American. soc pf trped p, 1997, 56: 44-52.

16.. Kilian A.H.D, Mughusu E.B, Kabagambe Sonnenbury F.V, comparison of two rapid, HRP-2 hased diagnostic tests for plasmodium falciparum. Trans of the R.S. of Trop. Med and Hyg 1997, 91: 666-667. 17. Perri GD, Olliaro P, Nardi S, Allegranzi B, Deganello R, Vento S, et al. The Parasight $\mathrm{F}$ rapid dipstick antigen capture assay for monitoring parasite clearance after drug treatment of plasmodium falciparum malaria, Trans of the R.S. of. Trop. Med and Hyg 1997, 91: 403-405. 